\title{
Incidence of Hyperprolactinemia in Infertile Women
}

\author{
Dr. Kalaivani, Dr. vidhyalakshmi \\ ${ }^{1,2}$ ( Medical officers - Tamilnadu $)$
}

\begin{abstract}
A prospective study was undertaken to determine the incidence of hyperprolactinemia in a group of infertile women, and to determine the association with abnormal menstrual function and presence or absence of galactorrhea. Among 100 infertile patients, 10(10\%) had elevated levels of serum prolactin. 2 (2.0\%) patients with hyperprolactinemia had menstrual irregularities and galactorrhea. With this study we found that hyperprolactinemia is a common finding in the infertile population, when they present with menstrual irregularities and galactorrhea.
\end{abstract}

Keywords: galactorrhea, infertility,, irregular menstruation, prolactin

\section{Introduction}

A. Hyperprolactinemia is defined as the elevation of prolactin levels above $25 \mathrm{ng} / \mathrm{ml}$ in women of child bearing age group and above $20 \mathrm{ng} / \mathrm{ml}$ in men and postmenopausal women [1]

Hyperprolactinemia is mainly due to enlargement of the pituitary gland or due to a pituitary tumour or can occur without any obvious reason(2)

Hyperprolactinemia is also frequently associated with secondary hypogonadotropic hypogonadism. This gonadotropic deficiency has been proposed to result from direct suppression of prolactin (PRL) on gonadotrophin-releasing hormone $(\mathrm{GnRH})$ release, but evidence supporting this mechanism has never been provided. PRL is synthesized and secreted by the lactotrope cells of the pituitary, and high levels of circulating PRL are mainly caused by lactotroph adenomas, which account for approximately $40 \%$ of all pituitary tumors. Pulsatile GnRH replacement can reverse hypogonadotropic hypogonadism and infertility induced by hyperprolactinemia in women as well as men $(3,4)$, suggesting that PRL excess in humans affects hypothalamic release of GnRH rather than directly affecting pituitary or gonad function.

\section{Effects Of Hyperprolactinemia}

Increased serum prolactin levels decreases the secretion of gonadotropin-releasing hormone ( $\mathrm{GnRH})$ in the hypothalamus, thus causing decreased secretion of LH and follicle-stimulating hormone (FSH) in the pituitary gland leading to decreased production of oestrogen and progesterone by the ovaries. (6)

Decreased hormone production in the ovaries leads to disruption of the normal follicular development causing atresia of the dominant follicle. (7)

\section{Etiology:}

Physiological increase in prolactin occurs during pregnancy; postpartum breastfeeding and stress.Prolactinomas account for 25 to $30 \%$ of functioning pituitary tumours and are the most frequent causes of chronic hyperprolactinemia. Other causes are non hypothalamic pituitary disease, polycystic ovarian syndrome, primary hypothyroidism, liver disease and chronic renal disease. $40 \%$ of patients of hypothyroidism have mild elevation of prolactin level. Some drugs like metoclopromide,

Phenothiazines, oestrogen and cimetidine can cause hyperprolactinemia. (8)

\section{Clinical Features:}

Often occur without symptoms.

\begin{tabular}{|l|c|}
\hline \multicolumn{1}{|c|}{ Presenting features } & Incidence \\
\hline Infertility (9) & $5 \%$ \\
\hline Galactorrhoea (10) & $20 \%$ \\
\hline Menstrual irregularities (10) & $30 \%$ \\
\hline Abortion (11) & $17 \%$ \\
\hline Decreased libido,hirsutism, acne & \\
\hline Pituitary adenomas- headache, visual field defects, external ophthalmoplegia (12) & $12 \%$ \\
\hline Progressive trabecular osteopenia- generalized body pain, low back ache. (13) & minimal \\
\hline
\end{tabular}


III. Method:

One hundred infertile women aged 18-35 years who suffered from infertility were selected from the infertility clinic for this study.

Prolactin level is measured in these women after obtaining history, short examination and informed consent.

The lab reference value for serum prolactin ranges from 2.8 to $29.2 \mathrm{ng} / \mathrm{ml}$.

\section{Results And Discussion:}

Of 100 infertile women, $10(10 \%)$ had elevated levels of serum prolactin, $2(2.0 \%)$ patients with hyperprolactinemia had menstrual irregularities and galactorrhea. With this study we found that hyperprolactinemia is a common finding in the infertile population more in the age group of 29-35 years when they present with menstrual irregularities and galactorrhea

\begin{tabular}{|c|c|c|c|c|c|}
\hline Pt ID & $\begin{array}{l}\text { AGE } \\
{[\text { In }} \\
\text { yrs }]\end{array}$ & $\begin{array}{l}\text { DURATION } \\
\text { OF } \\
\text { INFERTILITY }\end{array}$ & $\begin{array}{c}\text { TYPE } \\
\text { OF } \\
\text { INFERTILITY }\end{array}$ & $\begin{array}{l}\text { PROLACTIN } \\
\text { VALUE } \\
\left(\begin{array}{c}2.8-29.2 \\
\text { ng/ml })\end{array}\right. \\
\end{array}$ & SYMPTOMS \\
\hline 1. & 21 & 2 years & Primary & 14.7 & _- \\
\hline 2. & 21 & 2 years & Primary & 13.3 & - \\
\hline 3. & 22 & 3 years & Secondary & $\underline{37.8}$ & - \\
\hline 4. & 22 & 2 years & Secondary & $\overline{10.3}$ & - \\
\hline 5. & 22 & 2 years & Primary & 13.0 & - \\
\hline 6. & 22 & 4 years & Primary & 6.9 & _ \\
\hline 7. & 23 & 2 years & Primary & 8.8 & - \\
\hline 8. & 23 & 3 years & Primary & 31.4 & $\ldots$ \\
\hline 9. & 24 & 5 years & Primary & 15.2 & \\
\hline 10. & 24 & 4 years & Primary & 19.1 & - \\
\hline 11. & 24 & 2 years & Primary & 24.5 & - \\
\hline 12. & 25 & 3 years & Primary & 19.1 & _- \\
\hline 13. & 25 & 3 years & Primary & 9.9 & - \\
\hline 14. & 25 & 3 years & Primary & 24.7 & - \\
\hline 15. & 25 & 2 years & Primary & 11.1 & - \\
\hline 16. & 25 & 5 years & Primary & 10.6 & - \\
\hline 17. & 25 & 2 years & Primary & 12.9 & - \\
\hline 18. & 25 & 5 years & Secondary & 8.6 & - \\
\hline 19. & 25 & 2 years & Primary & 9.6 & - \\
\hline 20. & 26 & 1 year & Primary & 20.5 & - \\
\hline 21. & 26 & 6 years & Secondary & 25.1 & - \\
\hline 22. & 26 & 3 years & Primary & 17.2 & - \\
\hline 23. & 26 & 4 years & Primary & 26.4 & - \\
\hline 24. & 26 & 2 years & Primary & 16.7 & ${ }_{-}$ \\
\hline 25. & 27 & 5 years & Secondary & 18.2 & - \\
\hline 26. & 27 & 9 years & Primary & 43.8 & - \\
\hline 27. & 27 & 4 years & Primary & $\overline{11.5}$ & - \\
\hline 28. & 27 & 2 years & Primary & 13.3 & - \\
\hline
\end{tabular}

\begin{tabular}{|c|c|c|c|c|c|}
\hline 29. & 27 & 2 years & Primary & 16.8 & _ \\
\hline 30. & 28 & 7 years & Primary & 6.7 & $=$ \\
\hline 31. & 28 & 10 years & Primary & 10.7 & - \\
\hline 32. & 28 & 2 years & primary & 8.1 & - \\
\hline 33. & 28 & 1 year & Primary & 1.6 & - \\
\hline 34. & 28 & 5 years & Primary & 13.3 & - \\
\hline 35. & 28 & 6 years & Secondary & 4.2 & - \\
\hline 36. & 28 & 5 years & Primary & 11.2 & - \\
\hline 37. & 29 & 2 years & Secondary & 37.6 & - \\
\hline 38. & 29 & 2 years & Primary & 38.4 & 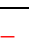 \\
\hline 39. & 29 & 4 years & Primary & 22.8 & - \\
\hline 40. & 29 & 6 years & Secondary & 15.9 & - \\
\hline 41. & 29 & 7 years & Primary & 28.3 & - \\
\hline
\end{tabular}


Incidence of Hyperprolactinemia in Infertile Women

\begin{tabular}{|c|c|c|c|c|c|}
\hline 42. & 29 & 2 years & Secondary & 11.7 & _ \\
\hline 43. & 29 & 1 year & Secondary & 11.2 & - \\
\hline 44. & 29 & 4 years & Primary & 16.0 & - \\
\hline 45. & 29 & 8 years & Primary & 20.5 & - \\
\hline 46. & 29 & 9 years & Primary & 23.2 & - \\
\hline 47. & 29 & 3 years & Primary & 22.9 & _- \\
\hline 48. & 30 & 10 years & primary & 17.6 & - \\
\hline 49. & 30 & 8 years & Primary & 4.1 & - \\
\hline 50. & 30 & 8 years & Secondary & 13.2 & - \\
\hline 51. & 30 & 6 years & Primary & 13.1 & - \\
\hline 52. & 30 & 4 years & Primary & 15.3 & - \\
\hline 53. & 30 & 2 years & Primary & 6.6 & - \\
\hline 54. & 30 & 2 years & Secondary & 11.6 & - \\
\hline 55. & 31 & 9 years & Secondary & 15.5 & - \\
\hline 56. & 31 & 4 years & Primary & 32.2 & $\begin{array}{c}\text { Galactorrhoea } \\
+\end{array}$ \\
\hline 57. & 31 & 9 years & Primary & 9.8 & - \\
\hline 58. & 31 & 3 years & Primary & 14.9 & - \\
\hline 59. & 31 & 8 years & Secondary & 15.2 & - \\
\hline 60. & 31 & 6 years & Secondary & 21.7 & - \\
\hline 61. & 32 & 8 years & Primary & 10.0 & _- \\
\hline 62. & 32 & 8 years & Secondary & 14.7 & - \\
\hline 63. & 32 & 12 years & Primary & 8.3 & - \\
\hline 64. & 32 & 5 years & Primary & 16.8 & _ \\
\hline 65. & 32 & 8 years & Primary & 14.6 & - \\
\hline 66. & 32 & 4 years & Primary & 41.8 & _- \\
\hline 67. & 32 & 13 years & Primary & $\overline{7.5}$ & - \\
\hline 68. & 32 & 3 years & primary & 15.8 & - \\
\hline 69. & 32 & 8 years & Primary & 16.1 & - \\
\hline 70. & 32 & 4 years & Primary & 21.7 & - \\
\hline 71. & 32 & 6 years & Primary & 15.7 & - \\
\hline 72. & 33 & 3 years & Primary & 21.1 & $=$ \\
\hline 73. & 33 & 4 years & Primary & 29.4 & $\begin{array}{l}\text { Menstrual } \\
\text { irregularity }\end{array}$ \\
\hline 74. & 33 & 8 years & Secondary & 7.4 & - \\
\hline 75. & 33 & 4 years & Primary & 9.8 & - \\
\hline 76. & 33 & 8 years & Primary & 21.3 & - \\
\hline 77. & 34 & 7 years & Primary & 12.7 & _- \\
\hline 78. & 34 & 5 years & Primary & 11.4 & - \\
\hline 79. & 34 & 7 years & Secondary & 8.3 & - \\
\hline 80. & 34 & 2 years & Primary & 5.3 & - \\
\hline 81. & 34 & 7 years & Secondary & 7.5 & - \\
\hline 82. & 34 & 10 years & Primary & 13.7 & 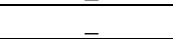 \\
\hline 83. & 34 & 6 years & Primary & 11.8 & - \\
\hline 84. & 35 & 4 years & Secondary & 8.2 & - \\
\hline 85. & 35 & 2 years & Primary & 10.1 & - \\
\hline 86. & 35 & 5 years & Primary & 5.2 & - \\
\hline 87. & 35 & 7 years & Primary & 18.2 & - \\
\hline 88. & 35 & 15 years & primary & 10.0 & - \\
\hline 89. & 35 & 6 years & Secondary & 8.5 & _ \\
\hline 90. & 35 & 10 years & Secondary & 9.5 & - \\
\hline 91. & 35 & 4 years & Primary & 8.3 & - \\
\hline 92. & 35 & 10 years & Primary & 35.3 & - \\
\hline 93. & 35 & 3 years & Primary & $\overline{25.5}$ & _- \\
\hline 94. & 35 & 11 years & Primary & 29.8 & 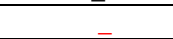 \\
\hline 95. & 35 & 8 years & Secondary & 18.9 & - \\
\hline 96. & 35 & 10 years & Secondary & 24.9 & - \\
\hline 97. & 35 & 3 years & Primary & 8.8 & - \\
\hline 98. & 35 & 4 years & Primary & 22.8 & - \\
\hline 99. & 35 & 5 years & Primary & 12.8 & - \\
\hline 100. & 35 & 3 years & Primary & 10.2 & _ \\
\hline
\end{tabular}

\section{Conclusion:}

Hyperprolactinemia is a common endocrine disorder in women presenting with amenorrhea and infertility and hence should be ruled out in all cases of amenorrhea and infertility as restoration of ovulation is seen in $90 \%$ of cases if cause of hyperprolactinemia is identified and treated.(14). 
Therefore whenever women presents with infertility, serum prolactin levels should be included in the routine protocol .

\section{References:}

[1]. Luciano AA, clinical presentation of hyperprolactenemia. J.Reprod Med 1999; 44 (suppl 12):1085-1090

[2]. Asa SL,Ezzat S. The pathogenesis of pituitary tmours. Nat Rev Cancer 2002; 2: 836-49

[3]. Bouchard P, Lagoguey M, Brailly S, Schaison G. Gonadotropin-releasing hormone pulsatile administration restores luteinizing hormone pulsatility and normal testosterone levels in males with hyperprolactinemia. J Clin Endocrinol Metab. 1985;60(2):258262. doi: 10.1210/jcem-60-2-258.

[4]. Lecomte P, Lecomte C, Lansac J, Gallier J, Sonier CB, Simonetta C. Pregnancy after intravenous pulsatile gonadotropin-releasing hormone in a hyperprolactinaemic women resistant to treatment with dopamine agonists. Eur J Obstet Gynecol Reprod Biol. 1997; 74(2):219-221. doi: 10.1016/S0301-2115(97)00091-2.

[5]. Billar BM,Luciano A.Crosignani PG,et al. Guidelines for the diagnosis and treatment of hyperprolactinemia. 1999. 44 (suppl12)); 1075-1084.

[6]. Hyperprolactinemia Michael O. Thorner, MD,MB $\quad$ BS, $\quad$ DSc, FRCP, David C. Harrison Medical Teaching Professor of Internal Medicine, University of Virginia Health System, Endocrinology and Metabolism, Box 801411, Charlottesville, VA 22908

[7]. Marcello D. Bronstein, MD, Professor of Endocrinology, Division of Endocrinology and Metabolism, Hospital da Clinicas, University of São Paulo Medical School, Av 9 de Julho 3858,São Paulo, SP Brazil The infertility manual , Kamini A Rao 3 rd Edition.

[8]. Samjahana Dhakal, The problem of infertility with hyperprolactenemia. NJOG 2010 Jul-Aug; 5(1): 7-11

[9]. Billar BM,Luciano A.Crosignani PG,et al. Guidelines for the diagnosis and treatment of hyperprolactinemia. 1999. 44 (suppl12)); 1075-1084.

[10]. Hyperprolactinemia and disorders of the menstrual cycle].Klinika za ginekologiju i akuserstvo Novi Sad, Medicinski fakultet.

[11]. Role of hyperprolactinemia in spontaneous abortion- Uma singh,S.Pandey, Sumith Arora, Anju mudra and Rajesh Arora.J.Biol.chem.Research.vol.26,No.1:1-6(2009).

[12]. Diagnosis and Treatment of Hyperprolactinemia: An Endocrine Society Clinical Practice Guideline.Cedars Sinai Medical Center (S.M.), Los Angeles, California 90048; University of Santiago de Compostela (F.F.C.), 15705 Santiago de Compostela, Spain; VA Palo Alto Health Care System (A.R.H.), Palo Alto, California 94304; New York University School of Medicine (D.L.K.), New York, New York 10016; Mayo Clinic Rochester (V.M.M.), Rochester, Minnesota 55905; University of Iowa (J.A.S.), Iowa City, Iowa 52242; and Churchill Hospital (J.A.H.W.), Headington, Oxford OX37LJ, United Kingdom

[13]. Forearm and Vertebral Bone Mineral in Treated and Untreated Hyperprolactinemic Amenorrhea*Departments of Internal Medicine and Radiology and the Clinical Research Center, University of Iowa Iowa City, Iowa 52242

[14]. Verhelst J,Abs R,Maiter D,et al. Cabergoline in the treatment of hyperprolactenemia : A Study in 455 patients. J Clin Endocrinol Metab 1999; 84: 2518-2522. 